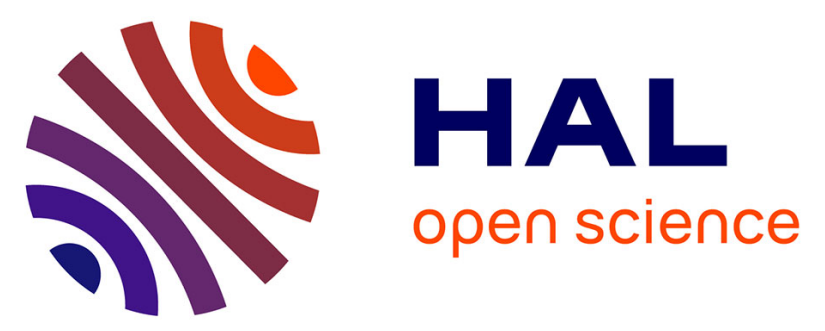

\title{
Challenges in recording and stimulation of living neural network based on original micro-electrode array (MEA) developments
}

Myline Cottance, Sebastien Nazeer, L. Rousseau, Gaelle Bazin Lissorgues, Sébastien Joucla, Blaise Yvert, Noëlle Lewis, Yannick Bornat, S. Renaud

\section{To cite this version:}

Myline Cottance, Sebastien Nazeer, L. Rousseau, Gaelle Bazin Lissorgues, Sébastien Joucla, et al.. Challenges in recording and stimulation of living neural network based on original micro-electrode array (MEA) developments. 2013 IEEE 11th International Conference on New Circuits and Systems (NEWCAS), Jun 2013, France. pp.1-4, 10.1109/NEWCAS.2013.6573645 . hal-00980781

\section{HAL Id: hal-00980781 https://hal.science/hal-00980781}

Submitted on 27 Jun 2014

HAL is a multi-disciplinary open access archive for the deposit and dissemination of scientific research documents, whether they are published or not. The documents may come from teaching and research institutions in France or abroad, or from public or private research centers.
L'archive ouverte pluridisciplinaire HAL, est destinée au dépôt et à la diffusion de documents scientifiques de niveau recherche, publiés ou non, émanant des établissements d'enseignement et de recherche français ou étrangers, des laboratoires publics ou privés. 


\section{Challenges in recording and stimulation of living neural network based on Original Micro-Electrode Array (MEA) developments}

\author{
M. Cottance, S. Nazeer, L. Rousseau, G. Lissorgues \\ ESIEE PARIS - ESYCOM EA2552 \\ University Paris Est \\ Noisy-le-Grand, France \\ g.lissorgues@esiee.fr
}

\author{
S. Joucla, B. Yvert \\ INCIA, CNRS UMR 5287 \\ University Bordeaux \\ Talence, France \\ N. Lewis, Y. Bornat, S. Renaud \\ IMS, CNRS UMR 5218 \\ University Bordeaux \\ Talence, France
}

\begin{abstract}
Recent research in micro-manufacturing together with new technologies in bioelectronics can lead to neuroscience applications to explore the properties of neural networks. Micro Electrode Arrays correspond to a well suited tool to either stimulate or record large populations of cultured cells and tissues. Based on the state of the art on MEAs, the paper describes original developments to improve recording or stimulation of neural networks, focusing on Action Potential discrimination.
\end{abstract}

\section{INTRODUCTION}

Emerging technologies in Bioelectronics can provide several fundamental and applied developments in medicine and biology. For instance extra cellular electrical recording of the central nervous system (CNS) has been used for decades by electro-physiologists to explore fundamental properties of neural networks.

Recent advances in micro and nano technology have opened the way to probe neuronal activity over large populations of neurons thanks to the fabrication of Micro Electrode Arrays (MEAs). Indeed, Micro Electrode Arrays (MEAs) are well suited for the stimulation and recording of neural cells as they correspond to a non-invasive technique allowing long-term stimulation and recording of bio-electrical signals of cultured cells and tissues [1]. In clinical an therapeutic research, such electrical stimulation of the CNS can be used in the field of neuro-prostheses to try to restore sensory or motor functions like in retinal [2, 3], cochlear [4] implants or in functional rehabilitation of upper and lower limb [5], in deep brain stimulation used in case of Parkinson disease [6]. In fundamental research, MEAs are now widely used as a tool to investigate neuronal networks to highlight their organization, connectivity, distribution and interactions between cells.
This paper will be divided in two main parts: i) the description of the operation principle of a MEA and its corresponding standard micro-manufacturing, ii) the description of several solutions to improve the action potential (AP) discrimination, which remains an important challenge in neuroscience applications.

\section{MEA OPERATION PRINCIPLE AND STANDARD MICRO- MANUFACTURING}

Multi-electrode arrays have been commonly used as a noninvasive in-vitro recording tool to study excitable cells and tissues, such as neurons. A typical MEA system includes four parts which are the following. First the MEA chip itself with its petri dish is used as a substrate to culture neuron cells and detect their electrical activity (spontaneous or stimulationinduced signals). Second, an amplifier circuit is required to filter and amplify the measured signals. Third, a specific stimulating circuit must be defined to apply either voltage or current impulses on specific electrodes to stimulate the cells located on top of them. Fourth, dedicated software is usually used to collect, store and analyze the acquired neural signals.

MEAs can be manufactured using micro-technology. The standard or classical manufacturing process consists in patterning metallic micro-electrodes with their leads using a lift-off method, on either a glass or an oxidized silicon wafer. For this purpose, the wafer is spin-coated with $2 \mu \mathrm{m}$ of NLOF 2020 (Clariant) photoresist, which is further removed at specific locations by photolithography to define the geometry of the metal layer (electrodes and tracks). In case of platinum MEAs, a first layer of $50 \mathrm{~nm}$ of titanium and a second layer of $150 \mathrm{~nm}$ of platinum are sputter-deposited over the whole 
wafer. The wafer is then placed in an ultrasonic bath with a dedicated remover AZ400, leaving only the metal layer deposited directly at the proper location. Then an insulation layer of silicon nitride (Si3N4) is deposited by PECVD (Plasma Enhanced Chemical Vapor Deposition) and further etched at the location of the electrodes, defining the electrode effective sizes. Si3N4 is etched using a $\mathrm{SF}_{6}$ plasma at locations not protected by the photoresist (i.e., electrodes). Finally, the wafer is cleaned and cut to separate the MEA chips and an annular glass ring is glued with poly-dimethoxy siloxane (PDMS) to constitute a recording chamber around the electrodes called the Petri dish.

The generic structure of the MEA chip is presented on Figure 1 , and the corresponding standard fabrication process is shown on Figure 2. The case of $3 \mathrm{D}$ electrodes will be described in the next section.

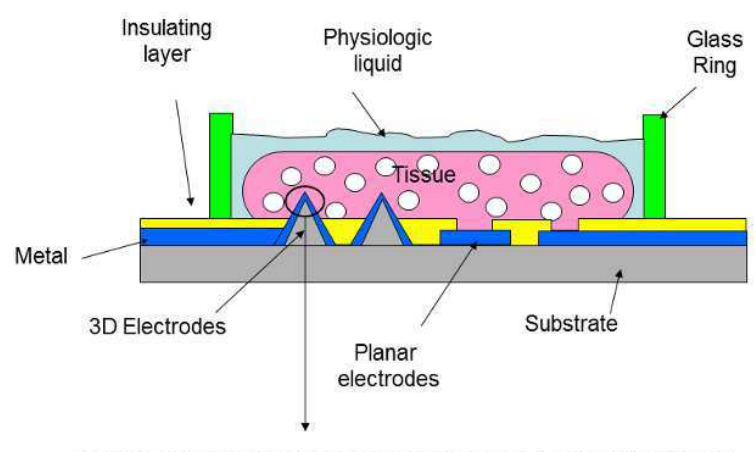

Metal / Liquid Interface : Helmholtz double layer capacitance

Figure1. Generic structure of the MEA chip

I) Oxydation of the silicon wafer

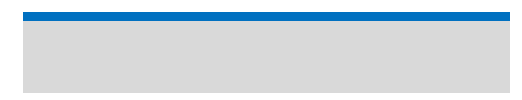

II) Metal deposition

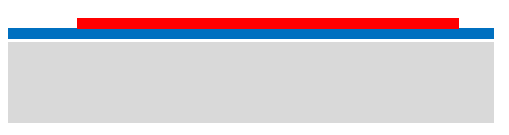

III) Si3N4 passivation layer

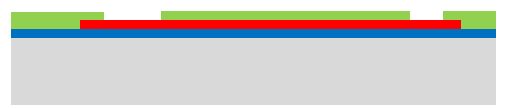

Figure2. Standard fabrication process of the MEA chip

\section{ACTION POTENTIAL DISCRIMINATION}

\section{A) High density MEAs and Multi-level recording}

One main interest of MEAs is to probe neuronal extracellular electrical activity at several sites simultaneously. In order to improve the localization of the potential fields recorded, it is necessary to increase the number of electrodes, leading to high density MEAs.
To reach this goal, one elegant solution is to define microelectrodes shaped as 3D needles instead of planar electrodes, at the tip of which is the recording site. The 3D shape will allow getting a very close contact to the cells [7].

In term of manufacturing constraints, the isotropic etching is classically used to etch silicon or glass substrates to process $3 \mathrm{D}$ electrodes, either with wet $(\mathrm{KOH})$ or dry (plasma) etching. But isotropic etching limits the electrode pitch regarding its height, preventing the fabrication of dense 3D electrode arrays with high aspect ratios. For example, a 3D electrode of $80 \mu \mathrm{m}$ height requires a minimum of $160 \mu \mathrm{m}$ pitch. Therefore, we developed MEAs with 64 (4x16), 256 $(8 \times 32)$ up to $1024(22 \times 47)$ recording sites using the Deep Reactive Ion etching (DRIE) technique with no limitations in height and diameter, as shown on Figure 3. Fabricated 3D electrodes of the MEA256 are micro-needles of $80 \mu \mathrm{m}$ in height, $250 \mu \mathrm{m}$ of pitch and lead tracks of $10 \mu \mathrm{m}$ in width.

Biological tests have been done to record activity from a whole embryonic mouse hindbrain-spinal cord, as illustrated on Figure 4.
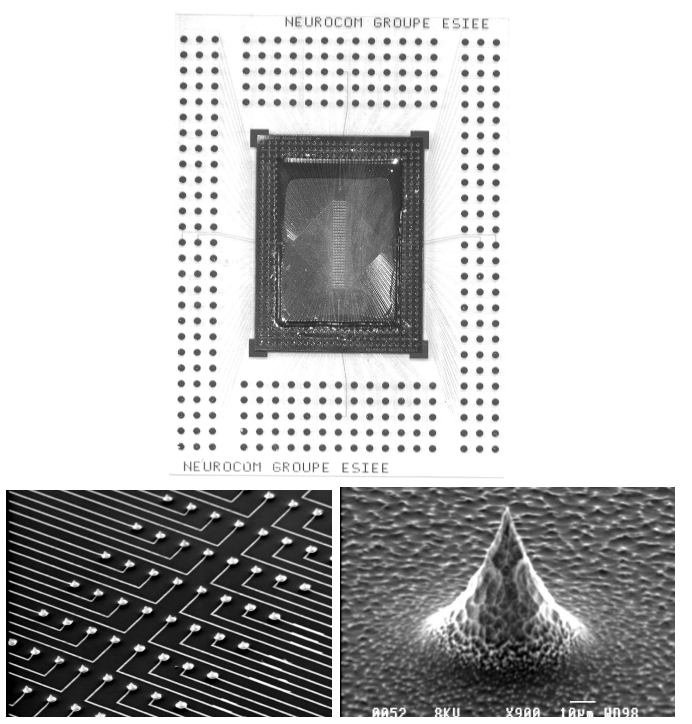

Figure 3. An example of a 256 Electrode array (MEA256) with 3D electrodes.
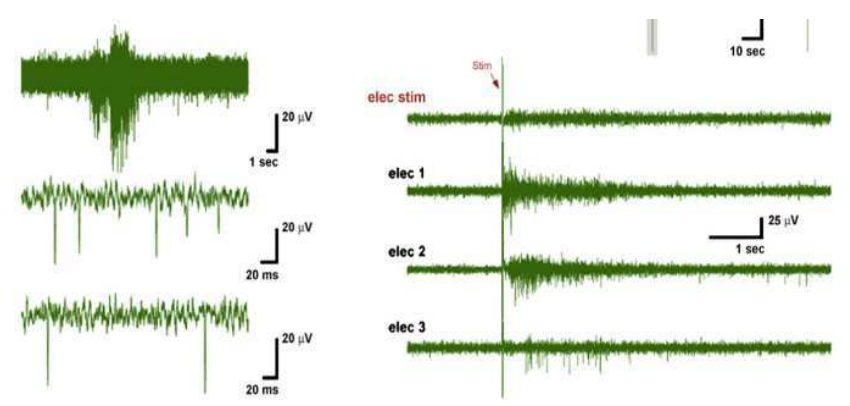

Figure 4. left) recorded activity; right) stimulated activity, on MEA256 - preparation on embryonic mouse spinal cord. 
As expected, increasing the number of recording sites allows new imagery of cellular information, with better discrimination of the involved neural cells, as on Figure 5. Indeed, we observe a loss of information in the case of 64 electrodes considered out of 256, missing a local response of the neural network (white arrow on the figure). Such images are obtained with specific signal processing for spatiotemporal mapping.

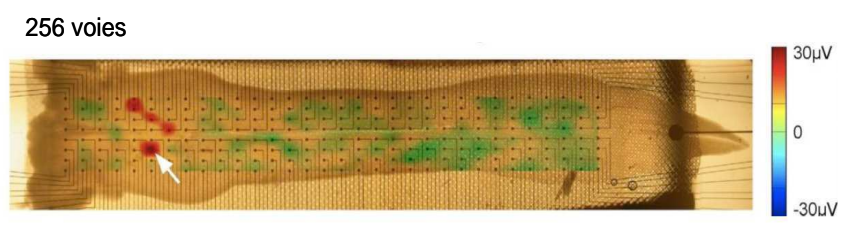

64 voies

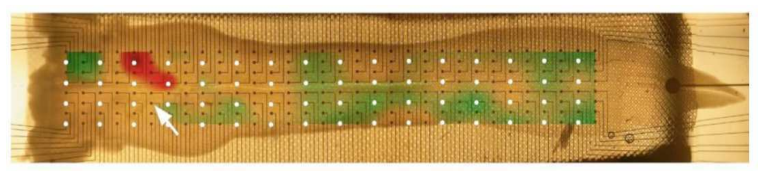

Figure 5. High density MEA for better discrimination.

Another solution to improve action potential discrimination is to increase the number of recording sites vertically in the depth of the tissues. Therefore we developed a new MEA chip as 32 shafts with 8 recording sites on each shaft, with a pitch of $50 \mu \mathrm{m}$. The technological process required to defined the shafts is then more complex due to a double side photolithography step followed by 2 DRIE steps (top and bottom), Figure 6.

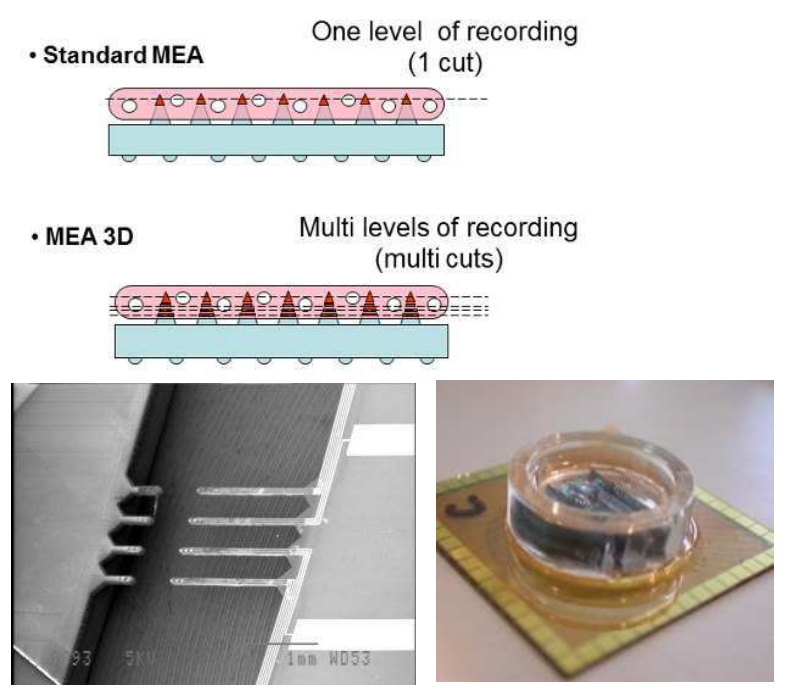

Figure 6. Principle of multi-level MEA ; fabricated chips

In both cases, either horizontal or vertical high density MEAs, the noise level depends on the electrode surface, as measured on Figure 7. The best available solution consists in increasing the effective electrode area while maintaining the diameter of electrode constant, which is possible with new materials like black platinum, titanium nitride or Iridium
Oxide. New developments are currently tested in this project with black platinum grown on the top of the electrodes.

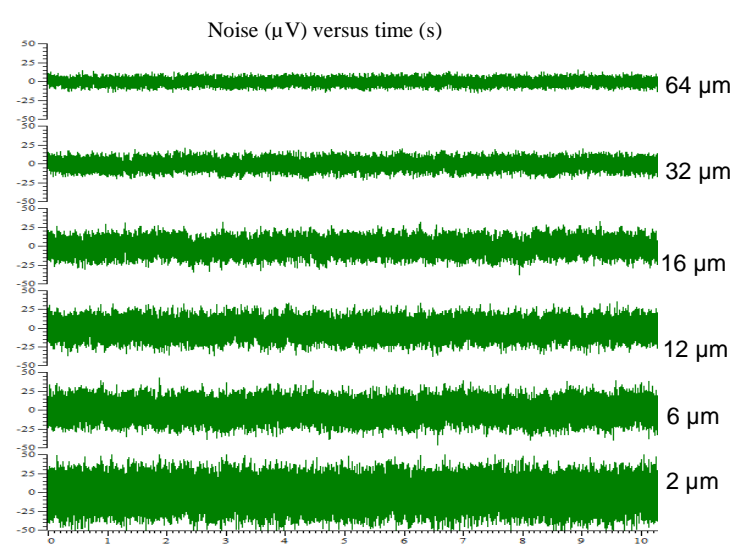

Figure 7. Noise level degradation with small electrode diameter.

\section{B) Dedicated signal processing}

An alternative strategy to improve action potential (AP) discrimination is to work on signal processing. Indeed, a solution for real-time AP detection is to implement a waveletbased pre-processor which emphasizes AP shapes and attenuates out-of-band noise [8]. The output of this preprocessor is used to compute an adaptive threshold and detect APs. The preprocessing algorithm is the Stationary Wavelet Transform (SWT), as wavelet filtering is performing in terms of compression and time-frequency localization for feature extraction. SWT algorithm has to be configured to ensure performing AP detection on the considered signal: the mother wavelet is closely related to the AP shape, the wavelet detail level is in accordance with the AP time frequency domain.

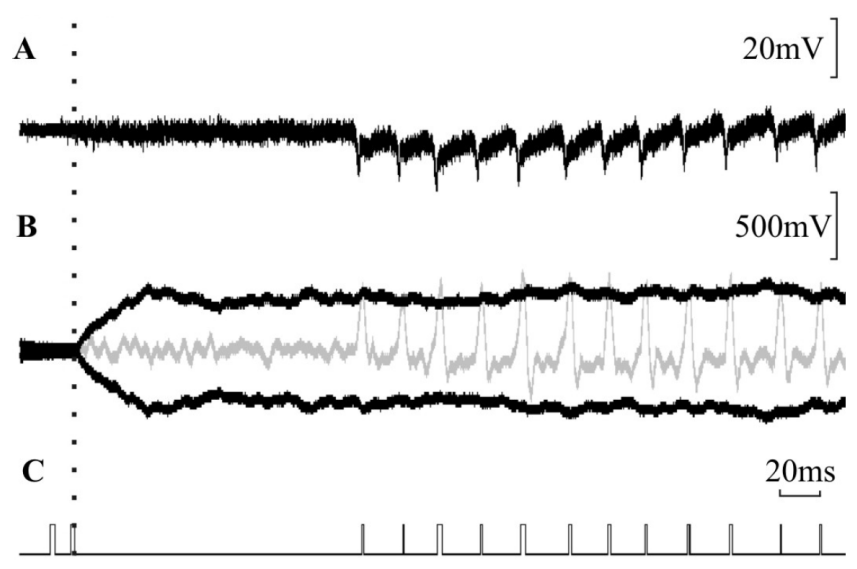

Figure 8. Example of wavelet based AP detection : A) original signal recorded from excitable cells cultured on commercial MEAs after filtering and digital conversion, B) SWT Sixth level detail output (grey) with threshold computed 
on the SWT first level detail output (black) C) Detected APs as digital output of the system.

\section{C) Focal stimulation}

In case of high density MEAs, one major problem is to avoid cross-talk between two or more electrodes in the stimulation mode.

One way to improve the focality is to force the stimulation current to flow back through a position close to the stimulating electrode. In the literature multipolar electrode configurations are generally considered $[9,10]$.

Hence we proposed and patented an alternative solution being to use a ground surface laying on the substrate of the MEA and surrounding all electrodes [11]. In that case, no additional external ground electrode is needed, since the whole current delivered by the stimulation electrode returns back through the ground surface. We observe on Figure 9 that, compared with the classical monopolar configuration, a better focality can be achieved with this configuration. Using a bipolar stimulation signal of $-/+2 \mu \mathrm{A}$ during $1 \mathrm{~ms}$ shows that the presence of the ground grid has divided the potential field by a factor of 2 .

Moreover, the potential field focality is more important when the surface conductance of the ground surface is high.
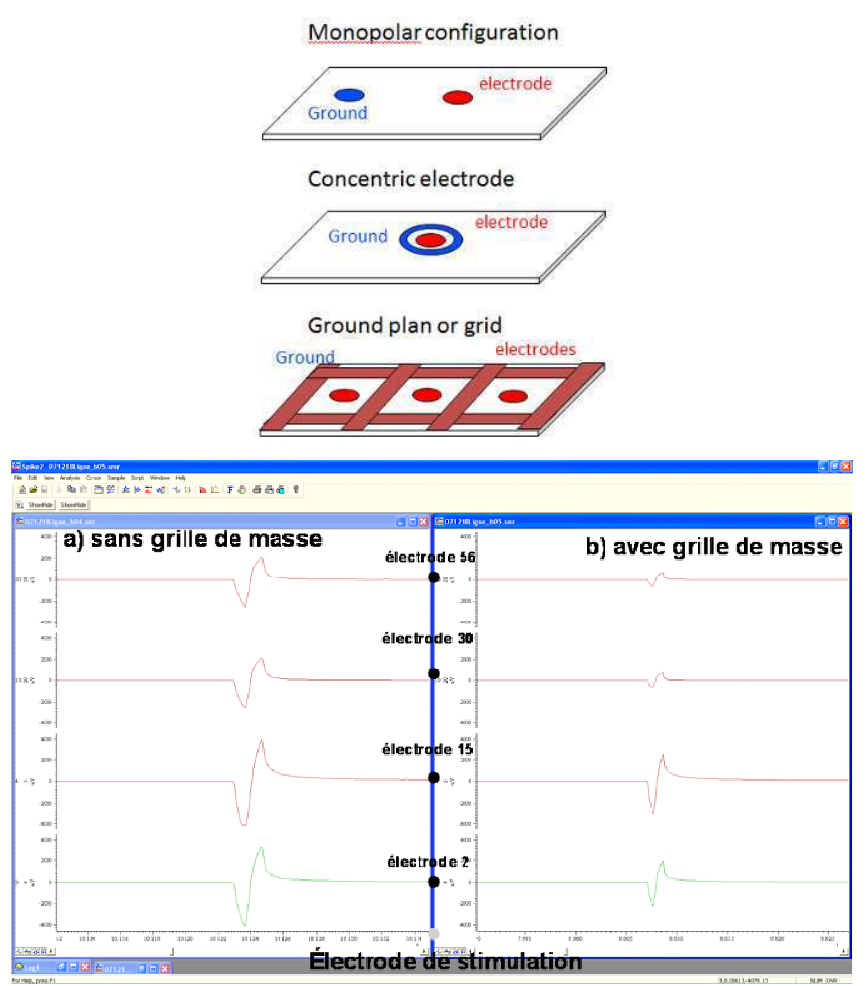

Figure 9. Ground grid focality improvement.

\section{ACKNOWLEDGMENT}

The authors would like to thank the French National Research Agency for their financial support through the ANR Blanc project HYRENE.

\section{REFERENCES}

[1] F. O. Morin, Y. Tkamura, E. Tamiya, "Investigating Neural Activity with Planar Microelectrode Arrays: Achievements and New Perspectives", vol. 100, N², pp. 131-143, 2005.

[2] J.-U. Meyer, "Retina implant - a bioMEMS challenge", Sensors and Actuators. A, Physical A 97-98, pp. 1-9, 2002.

[3] E.Margalit et al., "Retinal prosthesis for the blind," Surv. Ophthalmol, vol. 47, pp. 335-356, 2002.

[4] B. S. Wilson and M. F. Dorman, "Cochlear implants: Current designs and future possibilities," JRRD, Vol. 45, N ${ }^{\circ}$ 5, pp. 695-730, 2008.

[5] T. S. Grabow and al., "Spinal Cord Stimulation for Complex Regional Pain Syndrome: An evidence-based Medicine Review of the literature," The Clinical Journal of Pain, Vol. 19, No. 6, pp. 371-383, 2003.

[6] V. Voon and al., "Deep Brain Stimulation: Neuropsychological and Neuropsychiatric Issues", Movement Disorders, Vol. 21, Suppl. 14, pp. 305-326, 2006.

[7] A three-dimensional multi-electrode array for multi-site stimulation and recording in acute brain slices. Heuschkel MO, Fejtl M, Raggenbass M, Bertrand D, Renaud P (2002) J Neurosci Methods 114:135-148.

[8] A. Quotb, Y. Bornat, and S. Renaud. Wavelet transform for realtime detection of action potentials in neural signals. Frontiers in Neuroengineering, 4, 2011.

[9] Houweling, A. R. and M. Brecht, Behavioural report of single neuron stimulation in somatosensory cortex. Nature 451, pp.65-68, 2008.

[10] Holsheimer, J. and W. A. Wesselink, Optimum electrode geometry for spinal cord stimulation: the narrow bipole and tripole. Med Biol Eng Comput 35, pp.493-497, 1997.

[11] Joucla S, Yvert B (2009) Improved Focalization of Electrical Microstimulation Using Microelectrode Arrays: A Modeling Study. PLoS ONE 4(3). 